\title{
SOME THEOREMS RELATED TO A THEOREM OF E. HELLY ${ }^{1}$
}

\section{A. GLEN HADDOCK}

In 1957 Josef Molnar [1] proved the following theorem for the Euclidean plane: If in the plane an arbitrary number of simply connected, bounded and closed domains are given so that the intersection of every two is nonvoid and connected and the intersection of every three is nonempty, then the intersection of all the domains is not empty. Our Theorem 2 does not require that the intersection of every two be connected, and is a more general result than the result of Molnar. As a result of Theorem 1, Corollary 1 is equivalent to Molnar's result.

THEOREM 1. Let $\left\{C_{\alpha}\right\}$ be any collection of compact continua which do not separate the plane such that the intersection of any two is nonempty and connected. Then the union of any three fails to separate the plane if and only if the intersection of any three is nonempty.

This theorem follows in a straightforward manner from the Janiszvoski-Mullikin theorems.

COROLlaRY 1. If $\left\{C_{\alpha}\right\}$ is any collection of compact simply connected sets in the plane such that the intersection of any two is nonempty and connected, and the union of any three fails to separate the plane, then the intersection of the collection is nonempty.

This follows immediately from Theorem 1 , and the result of Molnar.

THEOREM 2. Let $\left\{C_{\alpha}\right\}$ be any collection of compact simply connected sets in the plane such that the intersection of any two is nonempty and the union of any three fails to separate the plane. Then the intersection of the collection is nonempty.

Proof. Assume that the union of some two sets $C_{1}$ and $C_{2}$ separates the plane. Let $D$ denote a bounded component of $S-\left(C_{1}+C_{2}\right)$. If $C_{\beta}$ is any other set of the collection, then $D \subset C_{\beta}$. Otherwise $C_{1}+C_{2}+C_{\beta}$ would separate the plane. Note that the boundary of $D$, denoted by $F$, is contained in $C_{1}+C_{2}$. It follows then from the Brouwer property that $F$ is connected [2]. Since $F=\left(F \cdot C_{1}\right)+\left(F \cdot C_{2}\right)$, it follows that

Received by the editors February 5, 1962 and, in revised form, May 31, 1962.

1 This work was supported in part by NSF grant G-9714 and constitutes a portion of the author's doctoral dissertation. 
$F \cdot\left(C_{1} \cdot C_{2}\right) \neq \varnothing$. Choose $p$ an element of the set $F \cdot\left(C_{1} \cdot C_{2}\right)$. Now since $F \subset C_{\beta}$ for every $\beta$, it follows that $p$ is an element of their intersection.

In the case that the union of no two sets separates the plane it follows that the intersection of any two sets is connected. The proof of the theorem is then completed by the use of Theorem 1.

\section{REFERENCES}

1. Josef Molnar, Über den zweidimensionalen topologischen Satz von Helly, Mat. Lapok 8 (1957), 108-114.

2. R. L. Wilder, Topology of manifolds, Amer. Math. Soc. Colloq. Publ. Vol. 32, Amer. Math. Soc., Providence, R. I., 1949.

Arkansas College and

OKLAHoma State University

\section{A NOTE ON EXACT SEQUENCES}

D. G. MALM

1. We consider a commutative diagram of abelian groups with exact rows :

$$
\begin{aligned}
& A_{1} \rightarrow A_{2} \stackrel{\beta}{\rightarrow} A_{3} \stackrel{\gamma}{\rightarrow} A_{4} \rightarrow A_{5} \\
& \downarrow \phi_{1} \quad \downarrow \phi_{2} \quad \downarrow \phi_{4} \quad \downarrow \phi_{5} \\
& B_{1} \rightarrow B_{2} \rightarrow B_{3} \rightarrow B_{4} \rightarrow B_{5}
\end{aligned}
$$

FIGURE 1

We suppose the $\phi_{i}$ are isomorphisms onto. The five lemma states that if $\phi_{3}: A_{3} \rightarrow B_{3}$ is a homomorphism for which the diagram (with $\phi_{3}$ inserted) commutes, then $\phi_{3}$ is an isomorphism onto.

Suppose $\eta_{1}, \eta_{2}: A_{3} \rightarrow B_{3}$ both give commutative diagrams, so that each is an isomorphism onto. (It is easy to find examples in which $\eta_{1} \neq \eta_{2 .}$.)

Proposition I. Under the above hypotheses,

$$
\eta_{1}^{-1} \eta_{2}(x)+\eta_{2}^{-1} \eta_{1}(x)=2 x
$$

for all $x \in A_{3}$.

To prove this, let $f=\eta_{1}^{-1} \eta_{2}$, and let $I$ be the identity function on $A_{\mathbf{3}}$.

Received by the editors March 13, 1962. 